\title{
Verificação em Hardware de Componentes de Comunicação
}

\author{
Maikel L. Kolling, Felipe A. Kuentzer, Cristiano Battisti, \\ Cristiano B. Both, Rafael R. dos Santos, Tatiana G. S. dos Santos \\ Departamento de Informática \\ Universidade de Santa Cruz do Sul (UNISC) \\ maikel@unisc.br, \{felipekuentzer, cristianobattisti\}@mx2.unisc.br, \\ \{cboth, rsantos, tatianas\}@unisc.br
}

\begin{abstract}
Resumo
Nas últimas décadas, a transformação nos meios $e$ formas de comunicação vêm impulsionando uma grande diversificação de dispositivos na área de comunicação de dados. Essa transformação é possivel, sobretudo, em decorrência do desenvolvimento da microeletrônica e dos sistemas embarcados. Apesar da grande evolução ocorrida nos equipamentos em si, ainda existe uma carência no que diz respeito aos testes e validações dos mesmos. Várias RFCs (Request for Comments) já foram escritas objetivando a definição de uma metodologia para benchmarking, mas a maioria são tipicamente implementadas através de software e os equipamentos para esse fim, disponiveis no mercado, possuem um alto custo agregado. Porém, com a necessidade crescente de aumento da vazão, muitos desses testes ficam restritos, já que o software não consegue atingir os requisitos de vazão e latência necessários. Assim, esse trabalho tem como principal objetivo implementar os testes descritos na RFC 2544 em hardware. Os resultados mostram que essa abordagem é bastante eficiente e flexivel, tendo em vista que não é necessário o uso de um sistema operacional e aplicativos de alto nivel.
\end{abstract}

\section{Introdução}

Atualmente, as redes de computadores transformaram-se em redes de informações que oferecem comunicação de dados, voz e vídeo, conhecidos como serviços triple play. As pessoas necessitam obter e trocar qualquer tipo de informação, a qualquer momento, em qualquer lugar. Isso alavanca o crescimento de provedores de serviço que oferecem conexões de banda larga. As tecnologias que são utilizadas para prover conexões, em geral, possuem um alto custo de implantação e necessitam constantemente serem atualizadas, tornando crescente $a_{\bullet}$ diversificação desses dispositivos.

Essa diversificação, embora importante na pesquisa e desenvolvimento de novos equipamentos, torna a padronização de procedimentos e protocolos uma necessidade comum na área. Assim, os fabricantes baseiam o desenvolvimento em normas, que descrevem as características técnicas dos dispositivos e protocolos de comunicação e garantem a conformidade dos equipamentos. Tipicamente, diversas normas e padrões são utilizados em todas as etapas do desenvolvimento, descrevendo os diversos níveis em que dada aplicação deve ser implementada.

Em decorrência da grande diversificação e do número de normas envolvidas, o desenvolvimento de testes de validação não é uma tarefa trivial. Atualmente os equipamentos de testes existentes no mercado possuem preços muito elevados. Conscientes desta carência em equipamentos de validação e na intenção de reduzir custos, muitos fabricantes validam e testam de forma manual e pouco detalhada apenas um pequeno percentual de sua produção. Uma validação por amostragem pode vir a mascarar algumas características técnicas dos dispositivos que não passaram por testes de conformação e desempenho [7].

Dentro desse contexto, inúmeras RFCs já foram descritas com a finalidade de formalizar os procedimentos de validação dos dispositivos de comunicação. Entre essas RFCs disponíveis, a RFC 2544 [6], estabelece um conjunto de testes que devem ser utilizados para melhor validar as características de desempenho de equipamentos de comunicação. Essa RFC, também, descreve como os equipamentos devem ser testados e como os resultados devem ser apresentados.

No entanto, nem todos os testes propostos são aplicáveis aos diversos tipos de dispositivos de comunicação, por não serem suportados pelo mesmo ou a sua 
irrelevância no escopo do dispositivo. Portanto, a seleção dos testes a serem utilizados na avaliação é de extrema importância. Adicionalmente, é fundamental um cuidado especial na análise dos resultados obtidos, exigindo uma repetição dos testes para uma futura validação estatística dos números obtidos [11].

Muitos testes propostos pela RFC 2544 são implementados em software. Porém, por necessitarem de um sistema operacional e serem executados como aplicação em um hardware não dedicado possuem desempenho limitado. Com essa motivação, este trabalho apresenta o projeto, implementação e validação de um protótipo em hardware para testes em redes Ethernet, baseado em um subconjunto proposto pela RFC 2544. A arquitetura desenvolvida possui blocos destinados a executar funções de geração de quadros Ethernet implementados em dispositivos reconfiguráveis do tipo FPGA (Field Programmable Gate Arrays), permitindo uma prototipagem rápida e de baixo custo através de uma linguagem de descrição de hardware [10].

Esse trabalho está organizado da seguinte forma: a Seção 2 mostra os trabalhos correlatos, enquanto que a Seção 3 apresenta maiores detalhes sobre os testes abordados e propostos pela RFC 2544. A Seção 4, por sua vez, apresenta o modelo da arquitetura de testes proposta e a Seção 5 mostra o ambiente utilizado nos experimentos efetuados. As Seções 6 e 7 apresentam, respectivamente, os resultados e as conclusões da pesquisa.

\section{Trabalhos Correlatos}

Apesar da grande quantidade de equipamentos de interconexão oferecidos no mercado, existe uma carência em equipamentos destinados a testes e validações para estes dispositivos. Normalmente, os equipamentos disponíveis implementados em hardware são extremamente caros. Os softwares disponíveis, por sua vez, não atingem as taxas requeridas por redes de alto desempenho. Desse modo, esse trabalho busca suprir essa necessidade mercadológica.

Um grande número de softwares foram desenvolvidos com a função de medir o desempenho em dispositivos de comunicação, destacando-se dentro dessa classe de softwares o Netperf [3] e o Iperf [2]. No entanto, esses softwares implementam apenas alguns dos diversos testes propostos pelas RFCs.

O software Netperf é uma ferramenta que foi desenvolvida pela Hewlett-Packard (HP), sendo que posteriormente teve seu código disponibilizado para a comunidade de software livre e atualmente possui versões para diferentes tipos de sistemas operacionais, entre eles Linux e Windows. O Netperf pode ser usado para medir inúmeros aspectos relevantes ao desempenho das redes de computadores, tais como vazão, latência e perda de quadros.

A principal característica desse software é a capacidade de realizar testes de transferência de grandes quantidades de dados, juntamente com a medição de desempenho de envio e recebimento de quadros. Assim, a ferramenta consegue medir a taxa máxima de transferência de dados do dispositivo em teste.

Para realizar estes testes o aplicativo envia durante 10 segundos rajadas contínuas de dados entre origem e destino, de acordo com configuração pré-determinada. Após, a ferramenta calcula o tempo gasto para enviar quadros de tamanhos fixos entre os dois nós que estão sendo analisados. Através do tempo de envio e do tamanho dos quadros enviados é possível determinar qual a banda total disponivel. Das ferramentas analisadas, o Netperf apresentou resultados mais precisos e por isso foi utilizada nesse trabalho, na etapa de validação, como será visto nas próximas seções.

Por outro lado, o número de dispositivos desenvolvidos em hardware destinados a testes de equipamentos de comunicação é bastante reduzido em relação aos softwares para esse mesmo fim. Os dispositivos em hardware de testes são normalmente utilizados em grandes empresas, em decorrência de seus preços muito elevados. Estes dispositivos, contudo, alcançam uma maior precisão nas medições de desempenho.

Um exemplo de dispositivo de teste é o Frame Scope Pro. Este equipamento, desenvolvido pela Agilent [4], é portátil e destina-se a testes em dispositivos de comunicação de alto desempenho, suportando taxas de transmissão entre 10/100/1000 Mbit/s. Esse trabalho, assim como o Frame Scope Pro, têm como objetivo testar componentes de interconexão. Porém, com um custo reduzido e com a possibilidade de realizar testes com caracteríticas diferentes do padrão Ethernet, como por exemplo gerar quadros com tamanhos menores e maiores para testar o comportamento de um dispositivo de interconexão. Esta possibilidade é devido a camada MAC (Media Access Control) estar implementada em um FPGA.

Outra pesquisa destinada ao estudo e integração de tecnologias em hardware e redes, diz respeito ao projeto NetFPGA [8]. Esse projeto, que possui um aspecto educacional, oferece uma plataforma em hardware específica para desenvolvimento de sistemas de comunicação de dados. Essa iniciativa prova o quanto essa área está em desenvolvimento e expansão. A plataforma NetFPGA, porém, não foi utilizada pois ela foi projetada de modo que a placa de desenvolvimento seja interligada em um PC hospedeiro através do barramento PCI. Essa pesquisa, no entanto, busca o de- 
senvolvimento de uma placa independente de outros equipamentos.

\section{Descrição dos Testes Abordados pela RFC 2544}

Quando o objetivo é a avaliação de equipamentos de comunicação, existem duas abordagens passíveis de serem realizadas: os testes de desempenho e os testes de conformidade. Os testes de conformidade têm como objetivo comprovar as reais características técnicas de um dispositivo de comunicação. Já os testes de desempenho visam quantificar o desempenho do equipamento em diferentes cenários de utilização. Com estas duas abordagens é possivel analisar diferentes equipamentos e determinar qual é o mais indicado para um determinado cenário [6].

A RFC 2544 propõe e define os testes que podem ser utilizados para descrever e comparar as características de desempenho dos dispositivos de interconexão de rede. Além disso, essa RFC informa o formato que os resultados dos testes devem ser apresentados. Essa seção apresenta e discute brevemente um sub-conjunto de testes de desempenho, descritos pela RFC 2544 e que fazem parte dos objetivos desse trabalho. São eles:

1. Throughput (Vazão): A vazão é definida pela RFC como sendo a quantidade máxima de dados que pode ser transportada da origem ao destino. Em qualquer sistema que utiliza o padrão Ethernet, a vazão máxima teórica é igual à taxa de dados, ou seja, $10 \mathrm{Mbit} / \mathrm{s}, 100 \mathrm{Mbit} / \mathrm{s}$ ou $1000 \mathrm{Mbit} / \mathrm{s}$. $\mathrm{Na}$ prática essas taxas não podem ser alcançados devido ao IFG (Inter Frame Gap), preâmbulo e SFD (Start Frame Delimiter). A vazão máxima, chamada neste trabalho de Vazão Ideal, é a quantidade de quadros que pode ser enviada por segundo, considerando o IFG, preâmbulo e SFD.

O teste de vazão é realizado através do envio de um determinado número de quadros com tamanho específico e uma taxa de transmissão prédeterminada. A RFC 2544 define que os quadros devem ser do tipo UDP (User Datagram Protocol) com tamanho entre 64 e 1518 bytes. A mesma RFC recomenda que valores para o tamanho do quadro sejam de $64,128,256,512,1024,1280$ e 1518 bytes. Em seguida, os quadros corretamente recebidos devem ser contabilizados. Se a quantidade de quadros recebido for menor que a quantidade de quadros transmitido uma menor taxa de transmissão deve ser selecionada e o procedimento de teste repetido.
2. Latency (Latência): O teste de latência diz respeito ao tempo total que o último bit de um quadro demora para chegar da origem ao destino. Entretanto, a origem e destino devem estar sincronizados. Esse tempo total é a soma dos atrasos do processamento nos elementos da rede somando ao atraso de propagação ao longo do meio de transmissão. Para medir a latência, a vazão deve ser inicialmente determinada, para todos os tamanhos de quadros definidos pela RFC. Posteriormente, uma série de quadros de teste, no mínimo 20 quadros, contendo uma primeira marca de tempo devem ser transmitidos pela rede. A marca de tempo é então verificada, pelo equipamento testador, no momento que os quadros são recebidos. Em seguida, é adicionada uma segunda marca de tempo nestes quadros. A latência pode ser calculada fazendo a diferença entre as duas marcas de tempo de cada quadro recebido. Uma outra forma de realizar o cálculo de latência é fazer com que os quadros de teste retornem ao equipamento testador através de um loop. $\mathrm{O}$ atraso é calculado utilizando-se a metade do valor sobre a diferença do tempo de ida e volta de cada quadro. Segundo a RFC 2544, a latência deve ser apresentada em microsegundos $(\mu s)$, por isso, todos os tempos apresentados neste trabalho utilizam essa grandeza.

3. Frame Loss Rate (Perda de Quadros): O teste de perda de quadros, por sua vez, é definido como sendo o número de quadros que foram transmitidos pela origem e não foram recebidos no destino. A avaliação de perda de quadros é importante para que seja verificado o comportamento do dispositivo testado em condições de sobrecarga da rede. Um exemplo dessa sobrecarga é quando são geradas rajadas excessivas de quadros em broadcast na rede.

O cálculo para taxa de perda de quadros é expresso em forma de porcentagem do número total de quadros transmitidos e segue uma equação fornecida também pela RFC. O procedimento para o teste resume-se na transmissão de rajadas constantes de quadros para cada taxa de vazão encontrada nos diferentes tamanhos de quadros. A primeira rajada de quadros é de $100 \%$ da vazão de cada tamanho de quadro, a segunda rajada de é $90 \%$ e assim sucessivamente até que chegue aos $10 \%$. Então, as taxas de perda são calculadas para cada porcentagem de rajadas enviadas no teste realizado.

4. Back-to-Back Frames: O teste de back-to-back frame diz respeito ao tamanho máximo de quadros contínuos que um dispositivo consegue tra- 
tar sem ter perdas de quadros ocasionadas por estouro de memória. Segundo a RFC 2544, o teste resume-se em enviar ao dispositivo uma rajada de quadros com um mínimo de espaço entre cada quadro. Tanto os quadros enviados como os quadros retornados devem ser contabilizados. Se o número de quadros recebidos for igual ao número de quadros transmitidos, é aumentada a duração da rajada e o teste é realizado novamente. Se o número de quadros recebidos for menor do que o número transmitido, a duração da rajada é reduzida sendo realizado novamente o teste.

5. System Recovery (Recuperação do Sistema): O procedimento definido como System recovery, por sua vez, tem como objetivo quantificar a velocidade que o dispositivo em teste reage a uma situação de sobrecarga de quadros. Ainda de acordo com a RFC 2544, uma rajada de quadros com cerca de $110 \%$ da vazão encontrada para cada tamanho de quadro deve ser enviada ao dispositivo em teste. Esses quadros devem ser enviados durante pelo menos 60 segundos, provocando assim um estouro de memória no dispositivo a ser testado. Assim que ocorrer o estouro de memória, o dispositivo em teste deverá parar de responder e então um tempo inicial de referência deve ser marcado. Após a marcação deste tempo inicial, uma nova rajada de quadros deve ser enviada ao dispositivo, porém a taxa de dados deve ser reduzida em $50 \%$. Uma nova marca de tempo deve ser registrada assim que o dispositivo voltar a responder aos quadros enviados. O tempo total necessário para que o dispositivo responda normalmente em caso de sobrecarga de dados é calculado subtraindo o tempo inicial pelo tempo final. O teste deve ser realizado algumas vezes para que uma amostragem estatística confiável seja coletada.

6. Reset (Reinicialização): Finalmente, o teste de reset tem como objetivo determinar o tempo necessário para o dispositivo em teste recuperar-se de uma reinicialização por hardware ou por software. O procedimento para o teste resume-se em transmitir um fluxo continuo de quadros ao dispositivo em teste. Em seguida, uma reinicialização por hardware ou por software no dispositivo em teste deve ser efetuado, registrando-se como tempo inicial o momento em que o último quadro foi recebido pelo equipamento testador. Assim que o equipamento testador passar a receber novamente os quadros do dispositivo em teste deve ser registrado outra marca de tempo. A diferença entre as duas marcas de tempo resultam no tempo que foi necessário ao dispositivo em teste se recuperar de uma reinicialização por hardware ou por software.

Para a validação da arquitetura desenvolvida este artigo apresenta um estudo de caso dos testes de vazão e latência, entre o grupo de testes definidos pela RFC 2544. A escolha foi devido aos testes serem os mais importantes para garantia de qualidade de serviço e para verificação da capacidade de transmissão do equipamento testado.

\section{Arquitetura de Testes Proposta}

Um sistema embarcado é um conjunto computacional dedicado que especializado e usa seu poder de processamento exclusivamente para a função a qual foi projetado. A utilização de dispositivos embarcados em plataforma do tipo FPGA mostra-se uma alternativa extremamente viável na implementação dos testes de vazão e latência, proposto pela RFC. Um FPGA é um dispositivo lógico programável de alta capacidade, chegando a integrar, em uma única pastilha de silício, milhares de células lógicas, além de núcleos de processamento RISC e memória RAM. Essas características, disponíveis nos atuais FPGAs, permitem uma implementação e prototipagem rápida com o uso de uma linguagem de descrição de hardware.

Desse modo, a arquitetura do ambiente de testes proposta, por esse trabalho, segue o fluxo de implementação em hardware através da tecnologia FPGA usando a linguagem VHDL (Very High Speed Integrated Circuit Hardware Description Language). As demais etapas de desenvolvimento como codificação, simulação, síntese, prototipação e validação foram assistidas por ferramentas automáticas disponibilizadas pela Xilinx [5] e Mentor Graphics [1]. Nas etapas de descrição, validação de código, sintese e geração de bitstream foi utilizado o ambiente de desenvolvimento Xilinx Integrated Software Environment (ISE) versão 9.1i, enquanto que a simulação e análise de cobertura foram efetuadas através da ferramenta ModelSim da Mentor Graphics.

A arquitetura proposta possui três componentes integrados ao Soft Core $M A C$ da empresa DATACOM [9], responsável pela comunicação com o meio físico (PHY). O Soft Core $M A C$ possui dois núcleos que desempenham o papel do protocolo MAC para redes Ethernet, capazes de suportar taxas de transmissão de 10/100 Mbit/s e $1000 \mathrm{Mbit} / \mathrm{s}$, respectivamente. Um terceiro núcleo possui a finalidade de prover enlace com o meio físico de fibra óptica. Esse Soft Core $M A C$, chamado no restante desse trabalho de MAC, é responsável pela implementação em hardware do subnível MAC do padrão Ethernet. Assim, a arquitetura desenvolvida 
que realiza a validação e testes de equipamentos de comunicação de dados é composta, por um gerador de quadros, um monitor de quadros e um gerador de reset. A arquitetura completa pode ser visualizada na Figura 1.

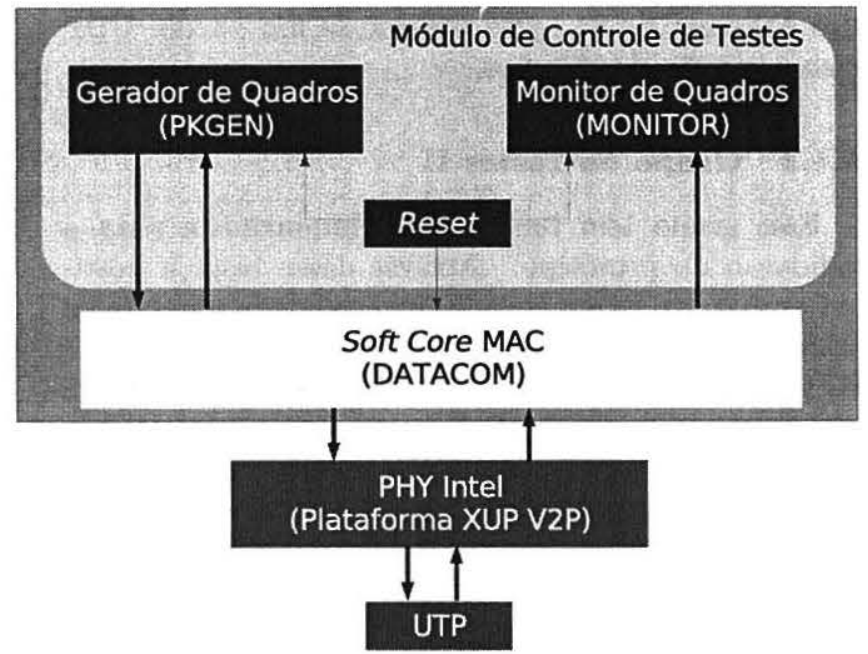

\section{Figura 1. Diagrama em bloco da arquitetura desenvolvida}

A versão inicial desse sistema prevê um ambiente de testes para redes Ethernet usando o MAC da DATACOM configurado para taxas de transmissão de 10/100 $\mathrm{Mbit} / \mathrm{s}$, half-duplex e full-duplex, seguindo o padrão IEEE 802.3. É importante salientar que a versão para taxas de transmissão de $1 \mathrm{Gbit} / \mathrm{s}$ já está em implementação. Porém, a arquitetura de testes é a mesma em ambas as situações.

Para a realização de testes é necessário a criação de um mecanismo que possui a função de gerar e gerenciar as operações básicas para o envio de quadros. Para isso, foi projetado o módulo gerador de quadros chamado PKTGEN. Esse componente possui a lógica necessária para o envio de quadros de diferentes tamanhos e características, seguindo as orientações da RFC 2455. O PKTGEN gerencia os sinais de controle do MAC e a captura das informações geradas pelo módulo monitor de quadros, informações que são incluídas nos quadros a serem transmitidos. A parte de controle é composta por uma máquina de estados, dividida em 5 estados, que têm por função interpretar os sinais originados do MAC e do módulo contador, para alternar entre seus estados. Através desta lógica é definindo a seqüência e forma de como os quadros são gerados.

O monitoramento de quadros, por sua vez, é responsabilidade do componente MONITOR. Esse módulo têm a função de identificar e contabilizar o número de quadros recebidos pelo MAC. Adicionalmente, esse bloco é responsável por implementar a lógica necessária para a captura das informações estatísticas já previstas no bloco MAC e por enviar os dados contabilizados para o bloco gerador de quadros.

A parte funcional resume-se em capturar as informações disponibilizadas pelo módulo estatístico do MAC e em cada ciclo contabilizar estes dados, incrementando os contadores que são disponibilizados para o módulo gerador de quadros. Outra função deste componente é capturar e disponibilizar ao módulo gerador de quadros, a posição de 4 chaves do tipo dip switch que determinam o tamanho do quadro a ser gerado.

O último bloco é responsável pela inicialização e sincronização dos demais blocos. Este componente contém a lógica necessária para a geração dos sinais de reset que são utilizadas no bloco gerador de quadros, MAC e MONITOR.

\section{Metodologia Experimental}

Essa Seção apresenta a metodologia para a validação do protótipo desenvolvido, que tem como objetivo analisar a conformidade e o desempenho de um dispositivo Ethernet. Após a completa implementação e validação do sistema, o trabalho voltou-se para os casos em que a arquitetura aqui proposta seria utilizada. Esses casos, aqui identificados como Cenários para Testes do Protótipo, serão discutidos na próxima Subseção.

O protótipo desenvolvido para testar equipamentos de comunicação, no padrão IEEE 802.3, possui quatro características fundamentais, importantes para a validação de suas funcionalidades. São elas:

- Capacidade de gerar tráfego com a taxa máxima de vazão, definido pela RFC 2544.

- Capacidade de gerar tráfego com tamanhos definidos pela RFC 2544.

- Capacidade de gerar quadros nos formatos especificados pela IEEE 802.3.

- Capacidade de medir quadros transmitidos e recebidos.

Desse modo, cada uma dessas características foram verificadas tanto através da validação funcional, como através da validação do protótipo após a sintese. Como já mencionado anteriormente, a etapa funcional foi realizada através do software ModelSim da Mentor Graphics. A parte de verificação já na placa, foi realizada através do software ChipScope da Xilinx.

Um exemplò de uma validação funcional com um quadro de tamanho mínimo constituído por: 7 bytes de 
preâmbulo, 1 byte de SFD, 6 bytes do endereço MAC de origem, 6 bytes do endereço MAC de destino, 2 bytes do tipo ou tamanho de quadro, 46 bytes de dados, 4 bytes de checksum e mais o intervalo entre os quadros (IFG) de 12 bytes, totalizando um total de 84 bytes. Sendo a freqüência de operação do bloco PKTGEN de $12,5 \mathrm{MHz}$, cada ciclo possui um período de $8.10^{4} \mu \mathrm{s}$, dessa forma, um quadro mínimo de 64 bytes deve ser enviado em 84 ciclos de $8.10^{4} \mu s$, totalizando um tempo de $6,72 \cdot 10^{6} \mu s$.

\subsection{Cenários para Teste do Protótipo}

Para finalizar a validação do protótipo foram realizados três diferentes grupos de testes. Primeiramente, realizou-se testes utilizando o software Netperf com o objetivo de analisar o desempenho alcançado em testadores implementados em software, para posteriormente compará-los com o protótipo desenvolvido. O segundo grupo de teste foi projetado com o objetivo de validar a capacidade máxima de geração de quadros pelo protótipo, isoladamente. Finalmente, o terceiro grupo teve a finalidade de analisar a vazão máxima e a latência alcançada em equipamentos de rede, demonstrando a eficiência do equipamento projetado.

Os recursos utilizados para a geração dos testes foram os seguintes:

- dois computadores Itautec com processador Athlon XP $1.8 \mathrm{Ghz}$, com $256 \mathrm{MB}$ de memória RAM, placa de rede 10/100 Mbits/s onboard e sistema operacional Linux;

- swicht 10/100, iniciado com as configurações de fábrica, com 24 portas padrão Ethernet, $64 \mathrm{Mb}$ de memória;

- analizador de protocolo Wireshark para capturar os quadros gerados pelo protótipo. Seu buffer foi configurado em $50 \mathrm{MB}$ para receber todos os quadros enviados pelo transmissor;

- cabos de interconexão, par trançado CAT 5e, com 1.5 metros de comprimento.

Todos os testes realizados para validação do protótipo, foram repetidos trinta vezes, com duração de dez segundos cada. A quantidade de repetições e o tempo de cada teste garante um intervalo de confiança de $99 \%$.

\subsubsection{Grupo de Testes I}

Para este grupo de testes foram utilizados dois computadores. Um computador em modo servidor e outro computador em modo cliente. No software Net- perf pode-se enviar pacotes UDP (User Datagram Protocol). Entretanto, o objetivo deste trabalho foi enviar quadros Ethernet com os tamanhos definidos pela RFC. Para isso, foi necessário considerar os cabeçalhos do protocolo IP ( 20 bytes) e UDP ( 8 bytes) no envio dos quadros pelo Netperf. Por exemplo, para um quadro Ethernet de 64 bytes utilizou-se um pacote UDP com tamanho de 18 bytes.

\subsubsection{Grupo de Testes II}

Esse grupo têm fundamental importância para a validação do protótipo. Através desse teste é possível avaliar, de forma isolada, a capacidade máxima de transmissão do protótipo segundo a recomendação da RFC. Para a sua realização, foi utilizado um cabo especial chamado de loopback. Este cabo foi confeccionado utilizando-se quatro condutores elétricos interligando os sinais de recepção com os sinais de transmissão em um mesmo conector. Com este cabo é possível enviar e receber quadros no protótipo que está sendo validado.

\subsubsection{Grupo de Testes III}

Para este grupo foram realizados testes com uma placa FPGA e a arquitetura prototipada. Esta placa estava conectada em um switch, que por sua vez, estava interligando a um computador para recepção e captura dos quadros.

Na próxima Seção é apresentada uma análise das taxas de vazão máxima alcançadas em comparação com a Vazão Ideal definida para um transmissão Ethernet de $100 \mathrm{Mbits} / \mathrm{s}$, bem como, as latências para diferentes tamanhos de quadros. Também, é descrito os resultados dos grupos de testes, demonstrando o desempenho do protótipo em relação aos testes realizados com software Netperf.

\section{Resultados}

O primeiro teste realizado teve como objetivo medir a vazão máxima alcançada pelo protótipo com um cabo loopback, como descrito no Grupo de Teste II. Os resultados obtidos podem ser visualizados na Figura 2, onde o eixo $\mathrm{X}$ representa os diversos tamanhos de quadro, definidos pela RFC e o eixo Y representa a quantidade de quadros enviados e recebidos por segundo. A curva da Vazão Ideal e a curva dos quadros enviados e recebidos pelo protótipo estão sobrepostas em todos os tamanhos de quadro. Com isso, demostrando que o protótipo desenvolvido têm a capacidade de gerar quadros utilizando $100 \%$ da capacidade de um equipamento Ethernet de $100 \mathrm{Mbits} / \mathrm{s}$. 


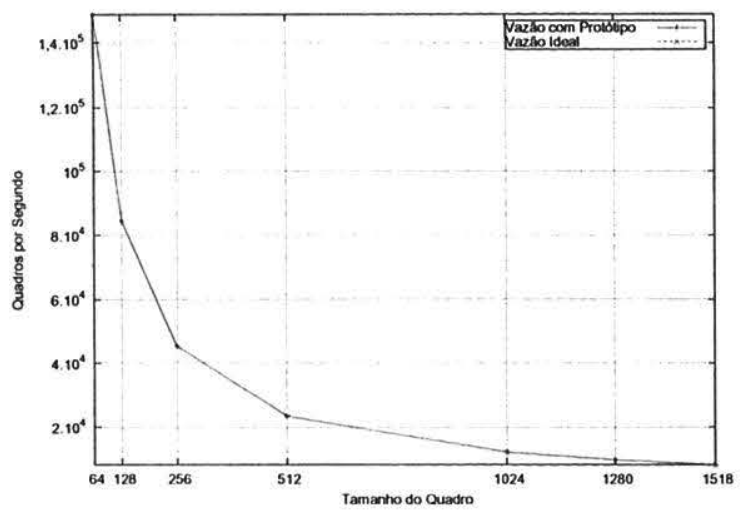

Figura 2. Teste com o protótipo em loopback

No Grupo de Teste II, também, foi realizado o teste da latência, conforme a RFC 2544. Para alcançar uma maior precisão nos resultados, utilizou-se uma freqüência de $100 \mathrm{MHz}$ para permitir a contabilização de ciclos da ordem de $0.1 \mu \mathrm{s}$. Um temporizador foi inicializado no momento que conclui-se o envio do último byte do quadro e a finalização do temporizador ocorreu no momento em que o último byte foi recebido pelo protótipo. Neste teste, a latência com o cabo loopback, para todos os tamanhos de quadro, foi de $0.25 \mu \mathrm{s}$, valor de ida e volta dividido por dois. A latência do protótipo para transmissão dos quadros foi extremamente baixa, entretanto devido a grandeza de $0.1 \mu s$ utilizada, os tempos para os diferentes tamanhos de quadros foram os mesmo.

O segundo teste realizado, utilizando os Grupos de Testes I e II, teve como objetivo comparar o desempenho do protótipo desenvolvido em relação ao software Netperf. Os resultados obtidos demonstram que em todos os tamanhos de quadro, o protótipo obteve um desempenho maior em relação Netperf. O desempenho do protótipo aumenta de forma acentuada para quadros menores. Este comportamento é justificado, pois o software Netperf necessita de um maior poder computacional para transmitir quadros menores. A Figura 3 apresenta as três curvas referentes a Vazão Ideal, a vazão alcançada com o Netperf e a vazão do protótipo.

Outra analise que pode ser realizada na Figura 3 é uma acentuada queda de desempenho para quadros de 64 bytes nos resultados utilizando o Netperf. Esta diminuição da vazão deve-se ao elevado uso de CPU pelo aplicativo no processamento dos cabeçalhos dos quadros Ethernet, saturando assim a capacidade de geração de novos quadros. Este elevado uso de CPU foi constatado durante a análise dos resultados do teste de

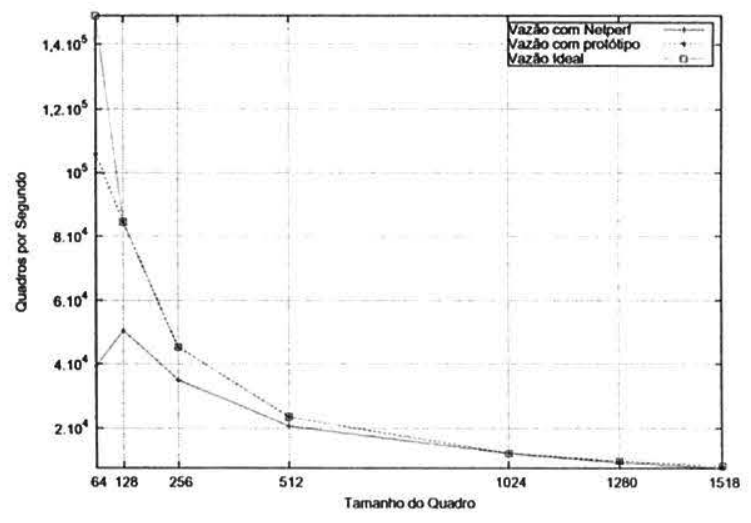

Figura 3. Teste da vazão entre computadores

vazão gerados pelo aplicativo Netperf.

No teste realizado com o protótipo, não foi possível alcançar a taxa máxima para quadros de 64 bytes. Alcançando-se uma vazão de $71 \%$ em relação a Vazão Ideal. Isso ocorreu devido a baixa capacidade de processamento da placa de rede onboard utilizada no computador que recebeu os quadros. A Figura 4 apresenta as curvas referentes as taxas de perdas de quadros obtidos nos testes dos grupos I e II. Estes valores mostram uma menor taxa de perda de quadros do protótipo em relação ao software.

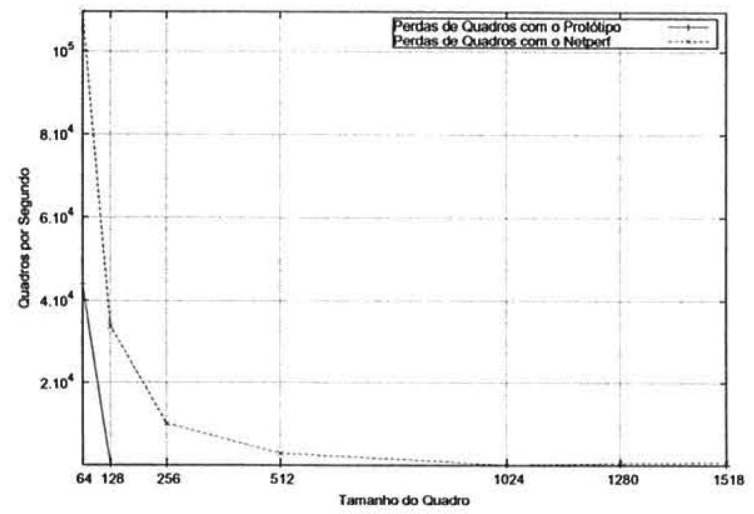

Figura 4. Quadros perdidos no teste da vazão

No Grupo de Testes III foi realizado o segundo teste da latência, conforme a RFC 2544. A metodogia seguiu a mesma configuração do primeiro teste da latência, descrito anteriormente. Entretanto, o loopback foi realizado no switch, que implementa o algoritmo de repassamento de quadros store-and-forward. Como pode 
ser observado na Figura 5, a latência encontrada neste teste aumentou de forma linear em relação aos tamanhos dos quadros transmitidos. A diferença da latência entre o maior e o menor quadro foi de $114.03 \mu \mathrm{s}$. Analisando-se a quantidade de bytes transmitidos por um quadro em relação a latência, os dois primeiros tamanhos de quadro (64 e 128 bytes) apresentaram um desempenho inferior aos demais. Por exemplo, a quantidade de bytes em relação a latência para quadros de 64 bytes foi de 0.175 , enquanto para quadros de 1.518 bytes a relação foi de 0.008 .

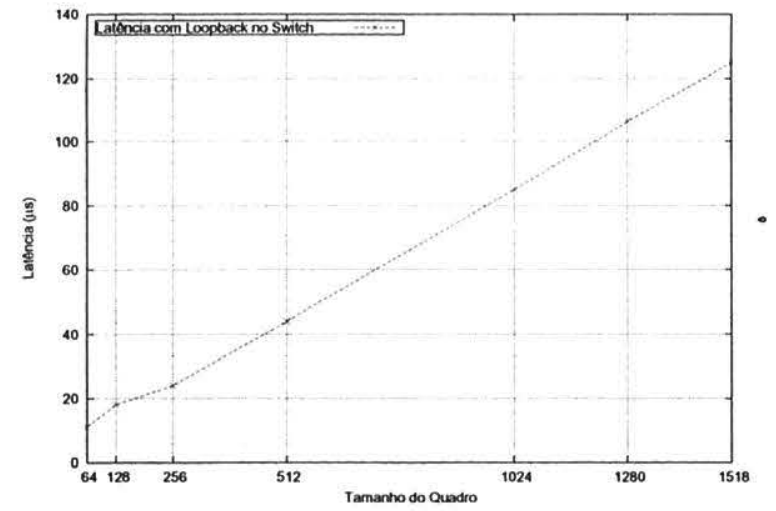

Figura 5. Teste da latência com loopback no switch

\section{Conclusões}

Este artigo apresentou uma arquitetura para testes em redes Ethernet utilizando um FPGA. A utilização desse hardware para os testes de vazão e latência obtiveram consideráveis ganhos de desempenho em relação aos testes realizado por software.

Nos testes realizados com o software Netperf verificou-se que existe um grande consumo de processamento para quadros de menor tamanho, assim a vazão medida foi inferior em relação as taxas teóricas de transmissão de quadros para tamanhos menores. Estes resultados são justificados, porque como os testes foram realizados em computadores de uso geral, o software não dispunha de todo poder de processamento para executar o teste. O ganho real de uma implementação em hardware foi apresentada neste trabalho, demostrando o desempenho em diferentes cenários de testes para equipamentos Ethernet.

O protótipo desenvolvido em hardware dispõe de todo poder de processamento do FPGA para a geração dos quadros Ethernet, possibilitando alcançar a Vazão
Ideal e uma menor latência na sua utilização. Além do desempenho superior o desenvolvimento do equipamento de teste em tecnologia FPGA, proporciona uma maior flexibilidade, permitindo a personalização dos blocos responsáveis pela geração de quadros Ethernet. Essa flexibilidade permite que se desenvolva trabalhos futuros, como por exemplo, a geração de quadros fora do padrão Ethernet, com objetivo de avaliar a conformidade dos equipamentos testados como a norma IEEE 802.3 .

\section{Agradecimentos}

Os autores agradecem o suporte oferecido na forma de bolsas, auxílios e equipamentos da UNISC e da empresa DATACOM.

\section{Referências}

[1] Modelsim SE user's manual. http://ww .model. com/resources/resources_manuals.asp. Mentor Graphics Corporation, June, 2006.

[2] Software Iperf. http://dast.nlanr.net/Projects/ Iperf/. June, 2008.

[3] Software Netperf. http://www.netperf.org. June, 2008.

[4] Agilent N2620A framescope pro network performance analyzer, January 2007.

[5] I. Avnet. Xilinx Virtex-II pro development kit, May 2003.

[6] S. Bradner and J. McQuaid. Request for Comments: 2544 - benchmarking methodology for network interconnect devices, 1999.

[7] S. Dibuz and P. Kremer. An easy way to test interoperability and conformance. In Proceedings of the 2nd International Conference on Testbeds and Research Infrastructures for the Development of Networks and Communities. IEEE, March 2006.

[8] G. Gibb, J. W. Lockwood, J. Naous, P. Hartke, and N. McKeown. NetFPGA an open platform for teaching how to build Gigabit-rate network switches and routers. IEEE Transactions on Education, 51, January 2008.

[9] C. Horna, F. Ramos, M. Barcelos, and R. Reis. Implementação e Validação de IP Soft Cores para Interfaces Ethernet 10/100 e 1000 Mbps Sobre Dispositivos Reconfiguráveis. In Proceedings of the 13th Workshop Iberchip, Lima, Peru, 2007.

[10] J. Moreno, P. Corrales, and J. Perez. Design of a parametrizable low cost Ethernet MAC core for SoC solutions. In Proceedings of the International Symposium on System-on-Chip. IEEE, November 2003.

[11] J. Talledo. Design and implementation of an ethernet frame analyzer for high speed networks. In Proceedings of the 15th International Conference on Communications and Computers, CONIELECOMP. IEEE, February 2005 . 